\title{
Una Experiencia de Aprendizaje por Proyectos Cooperativos. Visiones Docentes y Discentes
}

\section{A Learning Experience through Cooperative Projects. Teachers and Students Visions}

\author{
Blas González Alba * \\ Pablo Cortés González \\ Analia Leite Méndez \\ Universidad de Málaga, España
}

\begin{abstract}
La investigación que se presenta tiene como objetivo analizar y evaluar los resultados de un proyecto educativo, cooperativo e inclusivo desarrollado en un centro de Educación Primaria de la provincia de Málaga tras una formación en centro. Se ha hecho uso de estrategias de investigación como la observación participante, el análisis documental, los grupos de discusión y las entrevistas individuales al profesorado. De un modo general, en la investigación ha participado todo el profesorado del centro, asimismo, de un modo particular, hemos centrado la investigación en los grupos de $3^{\circ} \mathrm{A}$ y $4^{\circ} \mathrm{A}$ de Educación Primaria. Del proceso investigador han emergido dos categorías que giran en torno a las divergencias en enfoques y en la puesta en práctica del proyecto educativo, y las percepciones, valoraciones y experiencias del alumnado. Entre las conclusiones obtenidas resulta relevante señalar, por un lado, la aparición de tres perfiles docentes, caracterizados respectivamente por actitudes de indiferencia, de confusión e incompetencia ante la tarea, y de proactividad y motivación; y, por otro lado, encontrarnos con comentarios y valoraciones realizadas tanto por el profesorado como por el alumnado señalando los beneficios que les ha reportado la experiencia.
\end{abstract}

Descriptores: Educación primaria; Estrategia de aprendizaje; Innovación pedagógica; Aprendizaje cooperativo; Investigación cualitativa.

The aim of the research is to analyze and evaluate the results of an educational, cooperative and inclusive project developed in a primary school in the province of Malaga after training in the center. Research strategies such as participant observation, documentary analysis, discussion groups and individual interviews with teachers have been used. In a general way, all the teaching staff of the center have participated in the research, also, in a particular way, we have focused the research on the 3rd and 4th year groups of primary educations in the area of Spanish Language and Literature. From the research process, two categories have emerged that revolve around the divergences in approaches and in the implementation of the educational project, and the perceptions, evaluations and experiences of the students. Among the conclusions obtained, it is relevant to point out, on the one hand, the appearance of three teacher profiles, characterized respectively by attitudes of indifference, confusion and incompetence towards the task, and proactivity and motivation; and, on the other hand, to find comments and evaluations made by both the teachers and the students, pointing out the benefits that the experience has brought them.

Keywords: Primary education; Learning strategy; Educational innovation; Cooperative learning; Qualitative research. 


\section{Introducción}

A lo largo de los últimos años el aprendizaje cooperativo se ha posicionado como una de las principales estrategias innovadoras (Surian y Damini, 2014) que en el ámbito educativo ofrece una respuesta educativa e inclusiva al alumnado con necesidades específicas de apoyo educativo (Lata y Castro, 2015). Al respecto, son muchos y variados los estudios (Azorín, 2018; Gillies, 2014; Iglesias et al., 2017; Polo et al., 2017; Ribosa y Durán, 2018; Santos et al., 2020) que señalan los beneficios curriculares, sociales y emocionales que el uso de estructuras de aprendizaje cooperativo reporta a los estudiantes de un modo general, y al alumnado con necesidades específicas de apoyo educativo de un modo particular.

No obstante, la implementación en el aula de estrategias de aprendizaje cooperativo es un proceso complejo que requiere de formación específica tanto inicial (Sharan, 2010) como continua del profesorado (Angelides et al., 2007), así como de una actitud proactiva (Hijzen et al., 2007; Webb, 2009). Esta realidad ha supuesto que a lo largo de los últimos años se hayan invertido muchos esfuerzos para incorporar el aprendizaje cooperativo a la realidad del aula (Montanero y Tabares, 2020) y que, por lo tanto, se haya incrementado la oferta formativa de los Centros de Profesorado.

La presente investigación, que se contextualiza en el marco de una formación en centro en la que ha participado todo el profesorado y que ha tenido como objetivo que cada docente desarrolle un proyecto trimestral haciendo uso de una metodología cooperativa, es un ejemplo más de la predisposición en materia de formación que se viene desarrollando por parte de las administraciones educativas para incorporar el trabajo por proyectos cooperativos en las aulas. En este contexto y con el propósito de analizar la experiencia del profesorado en su proceso formativo y de implementación de un proyecto cooperativo se ha desarrollado la presente investigación.

\section{Aprendizaje por proyectos cooperativos}

Desde una mirada didáctica, hemos de considerar que la educación inclusiva encuentra en el aprendizaje cooperativo un aliado metodológico de primer orden. Como plantea Pujolás (2012), "la única manera de atender juntos en una misma aula a alumnos diferentes -tal como exige la opción por una escuela inclusiva- es introducir en ella una estructura de aprendizaje cooperativa" (p. 91), pues la educación inclusiva y el aprendizaje cooperativo, tanto en la teoría como en la praxis, son conceptos indisociables (Riera, 2011). En este sentido, son muchos los docentes que perciben la incorporación al aula de estrategias cooperativas como una respuesta viable hacia la inclusión efectiva (Lata y Castro, 2015; Riera, 2011), y la inclusión de lo cooperativo como elemento intrínseco al aula inclusiva (Azorín, 2018; Pujolás, 2012), pues permite al profesorado centrar los apoyos en los grupos o estudiantes que más lo necesitan mientras los demás se ayudan entre ellos (Montanero, 2019).

La incorporación de estrategias metodológicas y organizativas innovadoras, entre las que se encuentra el aprendizaje cooperativo, está estrechamente vinculada con la capacidad, necesidad y motivación del profesorado para mejorar la calidad de la enseñanza y ofrecer una respuesta educativa ajustada a la diversidad escolar. Entre las múltiples estrategias innovadoras, el trabajo por proyectos cooperativos se ha posicionado en los últimos años como uno de los procedimientos metodológicos de enseñanza más permeables a la 
atención a la diversidad y la inclusión (Pujolás 2012; Riera, 2011), ya que contribuye a generar prácticas educativas realmente transformadoras desde lo organizativo y didáctico y posicionan al alumnado en un rol activo (Loor et al., 2018) en sus procesos de aprendizaje desde la participación, la colaboración y el diálogo interpares.

El aprendizaje cooperativo hace referencia a múltiples métodos y técnicas (Johnson et al., 2013) bajo la lógica de la cooperación y el trabajo colectivo. No obstante, hay que considerar que el desarrollo de cualquier estrategia cooperativa requiere de la configuración de espacios, agrupamientos y tiempos más flexibles y heterogéneos (Cortés et al., 2018; Johnson et al., 2013; Riera, 2011). El trabajo cooperativo requiere que cada uno de los miembros del grupo tenga asignado un rol y unas funciones específicas (Domingo, 2008), y que el docente, en su papel multifacético, promueva el desarrollo de estrategias que potencien la interdependencia positiva, la interacción personal, la responsabilidad individual, la integración social y la evaluación grupal (Anderson, 2019; Johnson et al., 2013), facilitando la creación de espacios y tiempos que permitan al alumnado preguntar, discutir y rectificar (Pons et al., 2008).

Desde una dimensión organizativa y temporal, en el marco de la conformación de grupos de aprendizaje cooperativo, podemos encontrar grupos formales cuya composición dura entre una hora y semanas, informales con una duración que puede ser de minutos hasta una sesión de clase, o de base cooperativos que pueden durar casi un año (Johnson et al., 2013). Asimismo, las estrategias cooperativas se clasifican en: estructuras de aprendizaje simples, por ejemplo, Lápices al centro, el folio giratorio, la lectura compartida, y complejas, como por ejemplo, Técnica TAI (Team Assisted Individualization), el rompecabezas, la técnica TGT (Teamns-Games Tournament) (Pujolás, 2008).

En definitiva, como hemos evidenciado en otros trabajos (Cortés et al., 2018; GonzálezAlba, 2020), el aprendizaje cooperativo va a transformar la estructura organizativa y social del aula, potenciando el aprendizaje vicario, el trabajo conjunto, colaborativo y coordinado. Esta metodología, fundamentada en el constructivismo, permite que los estudiantes descubran y construyan nuevos aprendizajes (Riera, 2011), mejoren la capacidad de resolución de problemas y sus habilidades comunicativas y lingüísticas (Trujillo, 2002), la capacidad de liderazgo, de razonamiento crítico y la habilidad para escribir, dialogar y juzgar (Domingo, 2008) en un entorno potenciador de relaciones sociales (Mendo-Lázaro et al., 2018) que generan igualdad de oportunidades (Lata y Castro, 2015), fomentando el trabajo en equipo (Pujolás, 2012) y potenciando la creatividad (Catarino et al., 2019) desde la lógica de la diversidad como cualidad inherente a los procesos educativos.

Por último, cabe mencionar que el trabajo con estructuras cooperativas ha demostrado que contribuye a mejorar los resultados de aprendizajes, la motivación del alumnado (Gillies, 2016), la autoimagen (Santos et al., 2020), la empatía, la comprensión, la solidaridad (Martínez Lirola, 2016a; 2016b) y las habilidades sociales (Dyson et al., 2016; Gillies, 2014; Montanero y Tabares, 2020). Asimismo, y como exponen diferentes experiencias e investigaciones desarrolladas en educación primaria, a nivel curricular se evidencian mejoras en áreas como Matemáticas (Iglesias Muñiz et al., 2017; Ribosa y Durán, 2018), Lengua Castellana (Iglesias Rodríguez et al., 2017; Valdebenito y Durán, 2015), Lengua Inglesa (Cerdá y Querol, 2014; Polo et al., 2017), Ciencias Naturales (Moriña, 2011), Ciencias Sociales (Iglesias Rodríguez et al., 2017) o Educación Física (Prieto Saborit y Nistal Hernández, 2009). 


\section{Método}

La investigación se ha desarrollado en un colegio de Educación Infantil y Primaria de una línea que cuenta con una plantilla de 13 docentes y 176 estudiantes distribuidos en nueve unidades (3 de Educación Infantil y 6 de Educación Primaria). El centro educativo está ubicado en un pueblo de la provincia de Málaga con una población aproximada de 4000 habitantes.

La investigación se enmarca en el desarrollo de una formación con base en el centro realizada durante el curso 2018/19 y orientada a la implementación de estrategias cooperativas en el aula. Tras la finalización del proceso formativo (febrero de 2019), cada uno de los docentes ha desarrollado en su aula un proyecto en el que ha utilizado estructuras de aprendizaje cooperativo como tarea formativa final y ha recogido la experiencia mediante fotografías y reflexiones escritas como evidencias para justificar y valorar su implementación.

El objetivo principal ha sido conocer la actitud del profesorado ante la implementación de estrategias de aprendizaje cooperativo en el marco del diseño y desarrollo de un trabajo por proyectos. De un modo más concreto, los objetivos específicos han sido:

- Conocer la valoración que realizan los docentes sobre la puesta en práctica de estrategias cooperativas.

- Identificar las potencialidades, limitaciones y dificultades que ha encontrado el profesorado durante la implementación de estructuras de trabajo cooperativo.

- Analizar las valoraciones y comentarios del alumnado a lo largo de todo el proceso.

Para ello hemos planteado una investigación colaborativa y participativa (Hernández Rivero et al., 2020) que ha involucrado a todo el profesorado del centro, y de un modo particular, al alumnado de las aulas de $3^{\circ} \mathrm{A}$ y $4^{\circ} \mathrm{A}$ y a sus maestras tutoras, por ser las responsables de la implementación de las estrategias cooperativas en esos cursos. A partir de una muestra no aleatoria e intencional (Mayer, 2016) en la selección de los participantes se ha considerado (1) las clases con mayor número de alumnado diagnosticado con Necesidades Específicas de Apoyo Educativo (NEAE); (2) la voluntariedad de las maestras para participar en la investigación; y que (3) el horario del maestro de Educación Especial (observador participante) le permitiese participar activamente en la implementación y desarrollo de estrategias cooperativas junto a las dos maestras dentro de las aulas.

Con la intención de recoger la información en su contexto natural y de ser lo más respetuosos posible con las dinámicas cooperativas, la investigación se ha desarrollado en el aula ordinaria, como principal espacio de interacción escolar y aprendizaje. Se han empleado distintas técnicas de naturaleza cualitativa como la observación participante, el análisis documental (portafolio de grupo), los grupos de discusión de docentes y las entrevistas en profundidad al profesorado. A continuación, explicamos cada una de ellas con mayor detenimiento. 


\section{Cuadro 1}

Información de los dos grupos seleccionados

\begin{tabular}{lcclc}
\hline & Curso & Alumnado & \multicolumn{1}{c}{ Alumnado con NEAE } & Tutor/a \\
\hline Grupo A & $3^{\text {o E.P. }}$ & 26, 4 con NEAE & $\begin{array}{l}\text { Síndrome de Asperger; } \\
\text { Dificultad de aprendizaje por } \\
\text { discapacidad intelectual límite; } \\
\text { TDAH; dislexia. }\end{array}$ & Natividad \\
\hline Grupo B & $4^{\text {o E.P. }}$ & 23,5 con NEAE & $\begin{array}{l}\text { TDAH; } \\
\text { intelectual leve; Síndrome de } \\
\text { Asperger; dos alumnos con } \\
\text { dificultades de aprendizaje }\end{array}$ & \\
\hline
\end{tabular}

Nota. Elaboración propia.

Primero, la observación participante (Cohen et al., 2011) y el uso de un diario del investigador han facilitado la recogida de información. El doble rol de docente e investigador del primero de los autores le ha permitido observar y registrar -diario de campo- lo ocurrido en las aulas. Su uso ha tenido una doble finalidad, por un lado, ha servido como un documento de mejora y discusión de las prácticas desarrolladas, y, por otro lado, ha aportado información relevante para la investigación.

Segundo, el análisis documental (Gewerc y Vázquez-Calvo, 2020), en este estudio hemos analizado los 16 portafolios de grupo que han elaborado los estudiantes de los grupos de $3^{\circ} \mathrm{A}$ y $4^{\circ} \mathrm{A}$. Al respecto, hemos de considerar que el portafolio, como documento de reflexión y escritura permite analizar y reconstruir el trabajo realizado por el estudiantado (Jarauta y Bozu, 2013). Su análisis ha aportado información sobre las inquietudes, aprendizajes y dificultades que el alumnado ha plasmado al finalizar cada una de las sesiones. El portafolio se ha convertido en un instrumento de trabajo en el que los grupos han realizado sus tareas, escrito sus dificultades, registrado el trabajo diario, la autoevaluación de los aprendizajes adquiridos y su participación y sensaciones respecto del proyecto.

Tercero, de un modo general se han realizado tres entrevistas individuales semiestructuradas a los 13 maestros y maestras, estas han tenido una duración de unos 35 minutos y se han desarrollado durante los meses de septiembre (previa al inicio de la formación en centro), febrero y junio (tras finalizar la formación y la implementación de estrategias cooperativas, respectivamente). De un modo particular, las entrevistas individuales y en profundidad (Ruiz Olabuénaga, 1999) a las dos maestras-tutoras han tenido una duración media de 50 minutos. De las entrevistas a todo el profesorado han emergido una serie de cuestiones que giran en torno a (1) las limitaciones temporales de la propia formación en centro (tres sesiones de tres horas); (2) las dificultades experimentadas para diseñar y desarrollar el proyecto; (3) su percepción sobre las limitaciones y potencialidades que la implementación de estas estrategias tiene para el alumnado; (4) situaciones y percepciones personales y profesionales acerca de desarrollar o no estas estrategias.

Cuarto, a lo largo del curso se han mantenido dos reuniones mensuales de equipo de ciclo, estas son actividades de obligada asistencia para todo el profesorado (por ser actividades de coordinación docente amparadas en el plan de centro). En el caso que nos ocupa, tanto las dos maestras participantes como el maestro de Educación Especial, han formado parte del equipo de segundo ciclo de Educación primaria, y en el marco de estos encuentros se 
han desarrollado los grupos de discusión (Del Rincón et al., 1995). Estos encuentros se han desarrollado en nueve sesiones de 60 minutos -tres reuniones de ciclo por trimestreen dos momentos diferenciados y en los que han participado los tres miembros del equipo de ciclo. Durante la primera parte de la reunión se han organizado las tareas y actividades transversales y complementarias que competen de un modo oficial y profesional a este órgano de coordinación docente (ajenas a este proyecto, obligatorias, convocadas por la dirección del centro y que no han sido grabadas); en una segunda parte, y con el consentimiento oportuno de las dos maestras, se han grabado las aportaciones realizadas por los grupos de discusión. En estas sesiones han emergido temáticas similares a las que han emergido en las entrevistas al profesorado, de un modo particular estas han hecho referencia a (1) la complejidad de crear actividades cooperativas vinculadas con el proyecto que estaban desarrollando; (2) las potencialidades de esta metodología para contribuir al desarrollo de habilidades personales del alumnado; (3) la dificultad de diseñar, organizar y desarrollar estructuras cooperativas sin el apoyo de un segundo docente; y (4) la falta de tiempo para diseñar los proyectos.

A lo largo de todo el proceso se han considerado una serie de cuestiones éticas entre las que destacan que los docentes han participado de manera voluntaria, desinteresada y mostrando su consentimiento, que todo el procedimiento investigador (entrevistas y grupos de discusión) se ha desarrollado durante el periodo no lectivo y con el consentimiento del equipo directivo del centro, que se han empleado pseudónimos con el propósito de respetar y preservar el anonimato del profesorado y que tras la finalización y transcripción de las entrevistas y de los grupos de discusión se les ha devuelto las mismas al profesorado de un modo individual y/o grupal respectivamente. En este sentido, tras la finalización de todo el trabajo de campo se han transcrito las entrevistas y durante el mes de junio se han realizado las devoluciones individuales, se ha organizado un último grupo de discusión con el objetivo de leer la memoria final del proyecto (documento de centro) y de realizar una valoración general de la experiencia como parte del protocolo a seguir en la formación en centro.

En relación con el análisis de la información, cabe decir que posteriormente se ha procedido a la digitalización de la información contenida en el diario de campo y los portafolios. El proceso analítico se ha desarrollado en dos fases, una de "Codificación Abierta" y otra de "Codificación Axial" (Strauss y Corbin, 2002). Durante la primera etapa analítica el equipo investigador ha realizado un análisis del contenido, codificación de la información y un listado preliminar de categorías emergentes -descriptivas e interpretativas- de todos los documentos analizados.

En una segunda fase analítica se ha realizado lo que Strauss y Corbin (2002) denominan “codificación axial”, es decir, se han relacionado las categorías con subcategorías, de este proceso ha emergido el e esquema.

\section{Figura 1}

\section{Categorías analíticas}

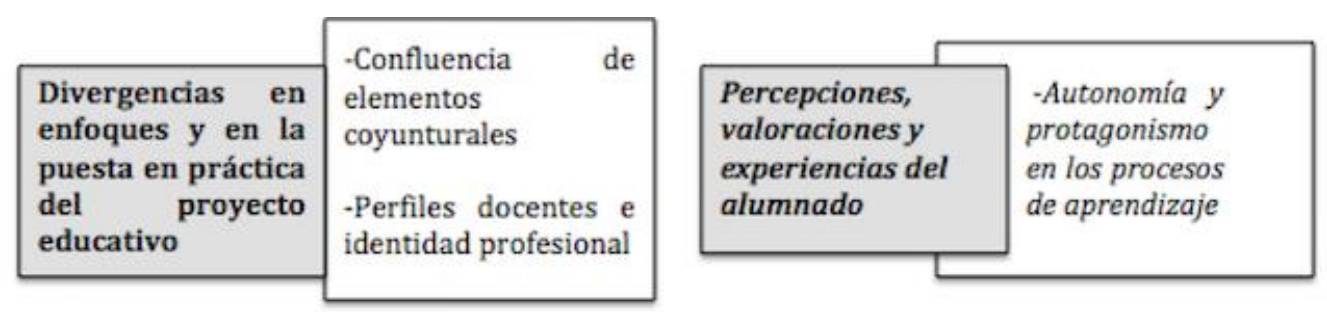


En los próximos epígrafes se describen las diferentes evidencias que se han obtenido a partir del proceso metodológico descrito. Como se desprende del árbol de categorías analíticas, éstas se relacionan, por un lado, con las actuaciones y percepciones del profesorado respecto a la promoción de la inclusión; y, por otro lado, con las valoraciones que el profesorado y el alumnado ha realizado de las diferentes experiencias cooperativas implementadas.

\section{Resultados}

\subsection{Divergencias en enfoques y en la puesta en práctica del proyecto educativo}

De las entrevistas al profesorado destacan discursos anclados en un paradigma escolar segregador en el que se evidencia una escasa formación docente en relación con la atención a la diversidad, insuficiente compromiso con una perspectiva educativa más inclusiva y tradiciones y discursos escolares vinculados con la exclusión escolar.

Creo que la atención especializada que puede tener un niño con necesidades fuera del aula es mejor y más individualizada, además que no molesta al resto. (entrevista a maestro de $5^{\circ}$ E.P.)

Estoy muy a favor de la inclusión, pero cuando el P.T. (maestro de educación especial) se lleva a David y a Lucas, la clase funciona mejor y pienso que a ellos también le viene mejor salir de clase. (entrevista a maestra de $2^{\circ}$ E.P)

Resulta interesante considerar lo que expresan ambos docentes (tutores) en relación con la atención educativa que recibe el alumnado con necesidades específicas de apoyo educativo al que atienden. La percepción de estos docentes acerca de cómo se debe desarrollar el trabajo de los especialistas en atención a la diversidad se ha revelado como un factor limitante en la implementación de estrategias cooperativas. En este sentido, nos encontramos con profesorado con destino definitivo en el centro desde hace más de una década, y que de un modo explícito también expresa su negativa a hacer uso de este tipo de estrategias.

To estoy haciendo algunas actividades puntualmente por el curso, en mi clase yo no puedo trabajar así, prefiero usar videos, explicar y que hagan las actividades. (entrevista a maestra de inglés)

Mis niños dentro de dos cursos van al instituto, no me veo haciendo esto el próximo curso porque tienen que ir preparados al instituto. (entrevista a maestro de $5^{\circ}$ E.P.)

Ahora mismo me cuesta mucho plantear algún proyecto, además no creo que sea la panacea para mejorar los resultados escolares. (entrevista a maestra de $2^{\circ}$ E.P)

Asistimos a discursos educativos arraigados y vinculados con una determinada cultura de centro de carácter histórico y en algunos casos segregador asociados con metodologías de enseñanza-aprendizajes tradicionales y transmisoras y compartidos por el profesorado más veterano del centro (4 maestros/as).

Por otro lado, encontramos un segundo grupo de docentes que aluden a una percepción de "incapacidad" para aplicar estas estrategias en sus respectivas aulas. Este hecho coincide con el profesorado cuya experiencia docente es menor de 5 años o mayor de 20 años y que no rechazan aplicar estas estrategias, pero que argumenta no disponer de formación $\mathrm{y} / \mathrm{o}$ competencias profesionales -recursos, estrategias y/o habilidades específicas- para implementarlas en sus respectivas aulas. 
Me está gustando la formación, pero a mi falta de experiencia se suma que nos están dando muchas actividades a la vez y yo me pierdo. No sé como aplicar lo que nos han enseñado con mis niños. (entrevista a maestra de Educación Infantil)

Veo muy bien todos los recursos que nos han dado, pero no sé como hacerlo en mi clase. Cuando he puesto a los niños a trabajar en grupo ha sido para hacer un trabajo y yo he ayudado a los que lo necesitan. (entrevista a maestra interina de $6^{\circ}$ E.P.)

Finalmente, encontramos a otro grupo de docentes que ha mostrado una actitud proactiva a lo largo del proceso formativo y de implantación de las estrategias cooperativas, y cuyos discursos hacen una valoración positiva de los beneficios que cualquiera de las estrategias utilizadas (trabajo por proyectos cooperativos y breakout) ha reportado de un modo general a sus respectivos grupos y, de un modo particular al alumnado diagnosticados con necesidades específicas de apoyo educativo, pues la formación del profesorado y la actitud docente respecto a la educación inclusiva son factores claves en su promoción (ColladoSanchis et al., 2020).

Cuando se les ha preguntado a las dos maestras de un modo explícito: ¿En qué ha modificado tu clase la incorporación de estrategias cooperativas en relación con la atención a la diversidad?, estas han destacado una mejora por parte de algunos estudiantes con necesidades educativas en el desarrollo de habilidades relacionadas con la competencia social.

He visto mejora en los exámenes, pero también en lo social, por ejemplo, con Samuel (Alumno con Síndrome de Asperger) ha mejorado su capacidad de trabajar en grupo, esto era impensable a principio de curso, también Juan (alumno con TDAH) que en clase no para y ha trabajado sentado bastante tiempo. (entrevista a tutora de $3^{\circ}$ de Primaria)

Me sorprende lo desenvuelta que está María (alumna con dificultades de aprendizaje), con lo que le cuesta leer en voz se está lanzando y también Salvador (alumno con síndrome de Asperger) se está empoderando, que es algo que le hacía falta. (entrevista a tutora de $4^{\circ}$ de Primaria)

Ambas docentes aluden constantemente a términos como "motivación" o "mejora curricular" cuando hacen referencia a los cambios percibidos en la clase. No obstante, y quizás el hecho más relevante está relacionado con el proceso de transformación experimentado por ambas maestras tras la formación y el cual les ha permitido concebir la inclusión como una acción colaborativa que les ha permitido modificar sus planteamientos metodológicos y organizativos y desarrollar estrategias cooperativas.

\footnotetext{
Me sorprende como están funcionando los grupos, también ayuda que tú (refiriéndose al maestro de PT) estés en clase conmigo, es más fácil de organizar. Veo a la clase muy motivada, aunque cuesta mucho trabajo organizar el breakout o las sesiones de trabajo cooperativo merece la pena. (Grupo de discusión, tutora de $4^{\circ}$ Primaria)

Como valoración general estoy satisfecha de cómo ha trabajado mi clase, me llevo muchos recursos para poner en práctica el año que viene, aun así, me cuesta plantear algo diferente a lo de este año. (Grupo de discusión, tutora de $3^{\circ}$ Primaria)
}

Los argumentos utilizados por el profesorado con respecto a la formación e implementación en sus respectivas aulas de estrategias cooperativas y/o favorecedoras de la inclusión, nos trasladan a tres grupos docentes y a tres escenarios educativos muy diferentes, cada uno de ellos vinculado con un modo de entender la atención a la diversidad, la inclusión y la incorporación de metodologías educativas innovadoras.

\subsection{Percepciones, valoraciones y experiencias del alumnado}


Una de las claves del trabajo por proyectos cooperativos es su carácter abierto, flexible y dinámico. Como se ha podido observar, este hecho ha permitido: (1) al alumnado, trabajar desde sus propios centros de interés, potenciando la motivación del alumnado al hacerlo responsable de su propio proceso de aprendizaje; (2) al profesorado, seleccionar aquellas estrategias cooperativas que mejor se adaptan a la edad, necesidades y capacidades del alumnado.

\begin{abstract}
Nuestro trabajo es de los planetas, lo hemos decidido con el 1,2,4, hemos investigado, después se las hemos explicado a nuestros compañeros y con la actividad lápices al centro hemos resuelto unas preguntas. (Diario de clase grupo 1 de $4^{o}$ de Educación Primaria)
\end{abstract}

Hemos hecho un cuento, entre todos hemos elegido un protagonista con la técnica 1,2,4, después con el folio giratorio cada uno ha escrito una cosa del protagonista, yo he dicho que era bajito, otro que tenía el pelo largo, otro los ojos azules, otro que iba vestido con la equipación del Madrid y otro que tenía una gorra, ha salido una cosa muy rara. (Diario de clase grupo 4 de $4^{\circ}$ E.P.)

Una cuestión central y que ha sido percibida en el aula ha sido la motivación que el alumnado muestra por el aprendizaje. La incorporación de estrategias manipulativas y creativas han permitido romper con las rutinas del aula y con la clase magistral, y han resultado ser un factor importante para el alumnado, de este modo lo ha recogido el maestro de Educación Especial en su diario: "cuando les he preguntado en diferentes sesiones al alumnado si prefiere trabajar así o en una "clase normal" la mayoría me contestan que prefieren los proyectos, aun así, me sorprende una minoría que prefiere trabajar en lo que ellos consideran una clase normal".

El aprendizaje por proyectos cooperativos ha sido combinado con otras estrategias, lo que ha facilitado que el alumnado desarrolle diferentes competencias. Del mismo modo, y como reflejan los diarios de clase, el alumnado ha asumido responsabilidades, ha desempeñado funciones asociadas a un rol $\mathrm{y}$ ha realizado tareas $\mathrm{y}$ actividades manipulativas y experienciales en grupo; es decir, ha desplegado destrezas, competencias y habilidades que de otro modo habrían resultado imposible.

\footnotetext{
Nos gusta mucho trabajar así porque cada uno tiene unas tareas y si uno no sabe ayuda al otro, también usamos el ordenador, buscamos las cosas, imprimimos, recortamos, pegamos y le decimos a la clase lo que hemos hecho. (Valoración grupal del proyecto, diario de clase del grupo 2 de $3^{\circ}$ Educación Primaria)

Lo mejor de los proyectos es que nos podemos ayudar y que cambiamos de grupo en cada proyecto. (Valoración grupal del proyecto, diario de clase del grupo 3 de $4^{\mathrm{o}}$ Educación Primaria)
}

El alumnado ha manifestado en sus respectivos diarios de campo muchos de los beneficios del trabajo por proyectos cooperativos. De un modo implícito, sus diarios aluden al compromiso, a la inclusión, a la responsabilidad para con el grupo, al aprendizaje por descubrimiento, a experiencias educativas compartidas, a la creatividad o a la imaginación. Sus reflexiones y valoraciones nos presentan espacios y oportunidades de aprendizaje que traspasan el currículum y que transitan hacia una escuela transformadora e inclusiva.

\title{
5. Discusión y conclusiones
}

A pesar de que la incorporación en el aula de estrategias de aprendizaje cooperativo contribuye a mejorar los procesos de inclusión (Pujolás 2012; Pujolás et al., 2013; Riera, 2011) la participación y protagonismo del alumnado en su proceso de aprendizaje (Orozco 
y Moriña, 2019), la implementación de estrategias cooperativas ha tropezado con estructuras microeducativas -actitud del profesorado- que están muy vinculadas con una cultura de centro y un discurso escolar segregador (González-Alba et al., 2020). Este hecho, que es más habitual de lo que pensamos, ha sido advertido por Domingo (2008), y se manifiesta cuando los centros y/o el profesorado pretenden incorporar y desarrollar estructuras cooperativas en las aulas y se encuentran con obstáculos políticos (del centro, del grupo...), culturales (costumbres, hábitos docentes, cultura de centro...) y/o técnicos (falta de espacios, escasa formación del profesorado, número de estudiantes...) que impiden o limitan su implantación.

De un modo particular, nos hemos encontrado con tres grupos docentes, (1) maestros y maestras que han mostrado actitudes de indiferencia hacia la incorporación de metodologías cooperativas; (2) docentes que se sienten confusos e incompetentes para desarrollar estas estrategias en sus clases; y (3) profesorado que ha mostrado una actitud proactiva y a los que tanto la formación experimentada como la implementación de estrategias cooperativas, les ha permitido ampliar su repertorio metodológico y promocionar prácticas inclusivas. Para comprender esta situación traemos a colación el trabajo realizado por De Boer y otros (2011), que han revisado 26 estudios internacionales que analizan las actitudes mostradas por el profesorado de Educación Primaria hacia la inclusión escolar. De esta investigación se desprende, entre otros resultados, que el profesorado con menor experiencia docente muestra actitudes más positivas hacia la inclusión y que la percepción de inexperiencia o falta de competencia para trabajar con alumnado con NEAE es otro factor limitante hacia la inclusión. En este sentido, nuestro trabajo arroja resultados similares, tal y como hemos manifestado anteriormente.

$\mathrm{Al}$ respecto, la literatura especializada (Catarino et al., 2019; Domingo, 2008; Johnson et al., 1999; Lata y Castro, 2015; Pujolás, 2012; Riera, 2011) hace referencia a los muchos beneficios que el aprendizaje cooperativo reporta al alumnado en su desarrollo competencial, personal, social y curricular. No obstante, la presente investigación también coincide con los resultados obtenidos por el estudio desarrollado por Jenkins y otros (2003) tras investigar las prácticas cooperativas desarrolladas por 21 docentes. En este sentido, los autores destacan que la incorporación de estructuras de aprendizaje cooperativo de un modo regular a la rutina del aula ha mejorado el autoconcepto, el sentimiento de pertenencia al grupo y una mejora curricular, es decir, dimensiones intrínsecas a la escuela inclusiva y que se repiten en el discurso del profesorado que ha participado en esta investigación e implantado estrategias cooperativas.

A modo de conclusión, y considerando las cuestiones que venimos señalando y los resultados arrojados por esta investigación, la implementación de estrategias cooperativas requiere de:

- Un proceso formativo complejo y dilatado (formación inicial y continua) de todo el profesorado que pretenda incorporar estas estructuras de aprendizaje en su aula y transformar la cultura de centro (Escudero y Martínez , 2011) pues, como señalan Gillies (2014), Buchs y otros (2017) y Veldman y otros (2020), la actitud y creencia del profesorado hacia el aprendizaje cooperativo son los factores que van a contribuir a su implementación efectiva y los que van a favorecer la creación de una cultura inclusiva, de una política inclusiva y de prácticas inclusivas (Crisol, 2019). 
- El apoyo y disposición de al menos un/a docente y el maestro/a especialista, cuya función en este contexto es fomentar la participación y aprendizaje del alumnado más vulnerable (Pujolás et al., 2013) y atender en un contexto inclusivo al alumnado con necesidades específicas de apoyo educativo que tiene asignado.

- Una transformación de las lógicas que subyacen a los procesos de enseñanzaaprendizaje, esto es, el profesorado ha de estar dispuesto a ceder el protagonismo al alumnado (Pons y otros 2008) a transformar la organización y los espacios del aula (Cortés González et al., 2018) y a promover la diversidad de formas de aprender, ser y estar en el aula (Duk y Murillo, 2016) diseñando proyectos que faciliten la participación activa de todos los estudiantes.

En definitiva, somos conocedores de las limitaciones de este trabajo, principalmente las relacionadas con la cantidad de profesorado que ha participado en la investigación, con la dificultad y limitación temporal de ampliar la presencia de los investigadores como observadores participantes en más aulas y con la necesidad de hacer partícipe al alumnado en la investigación más allá de considerar las valoraciones que han realizado en sus respectivos portafolios. No obstante, y considerando los resultados obtenidos, como investigadores hemos de seguir avanzando en la investigación de experiencias escolares que implementen prácticas cooperativas. En este sentido, sabemos que estamos ante un reto que requiere de formación inicial y continua, de mayor disponibilidad de recursos humanos, de procesos de planificación y de una organización escolar que permitan rediseñar los espacios y tiempos tradicionales del aula. En definitiva, precisamos de una amplia transformación que nos permita construir una escuela comprensiva e inclusiva en la que la participación y cooperación de todos su agentes educativos y escolares sea un principio que vehicule la enseñanza y el aprendizaje con expectativas, posibilidades y realidades de la comunidad educativa. Para ello es necesario dirigir la formación docente, inicial y continua, hacia una cultura escolar inclusiva y metodologías colaborativas y cooperativas.

\section{Referencias}

Anderson, J. (2019). Cooperative learning: Principles and practice. English Teaching Professional, $121,4-6$.

Angelides, P., Stylianou, T. y Leigh, J. (2007). The efficacy of collaborative networks in preparing teachers. European Journal of Teacher Education, 30(2), 135-149. https://doi.org/10.1080/02619760701273953

Azorín, C. (2018). El método de aprendizaje cooperativo y su aplicación en las aulas. Perfiles Educativos, 4O(161), 181-194. https://doi.org/10.22201/iisue.24486167e.2018.161.58622

Baines, E., Blatchford, P. y Kutnick, P. (2008). Pupil grouping for learning: Developing a social pedagogy of the classroom. En R. M. Gillies, A. Ashman y J. Terwe (Eds.), The teacher's role in implementing cooperative learning in the classroom (pp. 56-72). Springer.

https://doi.org/10.1007/978-0-387-70892-8_3

Blanco, R. (2006). La equidad y la inclusión social: Uno de los desafíos de la educación y la escuela hoy. REICE. Revista Iberoamericana sobre Calidad, Eficacia y Cambio en Educación, 4(3), 1-15. 
Buchs, C., Filippou, D., Pulfrey, C. y Volpé, Y. (2017). Challenges for cooperative learning implementation: Reports from elementary school teachers. Journal of Education for Teaching, 43(3), 296-306. https://doi.org/10.1080/02607476.2017.1321673

Catarino, P., Vasco, P., Lopes, J., Silva, H. y Morais, E. (2019). Cooperative learning on promoting creative thinking and mathematical creativity in higher education. REICE. Revista Iberoamericana Sobre Calidad, Eficacia y Cambio en Educación, 17(3), 5-22. https://doi.org/10.15366/reice2019.17.3.001

Cerdá, C. V. y Querol, M. J. (2014). El aprendizaje cooperativo para el desarrollo de la competencia comunicativa oral en lengua inglesa en el aula de primaria. Encuentro. Revista de Investigación e Innovación en la Clase de Idiomas, 23, 16-29.

Cohen, M. L., Manion, L. y Morrison, K. (2011). Research methods in education. Routledge.

Collado-Sanchis, A., Tárraga-Minguez, R., Lacruz-Perez, I. y Sanz-Carvera, P. (2020). Análisis de actitudes y autoeficacia percibida del profesorado ante la educación inclusiva. Educar, 56(2), 509-523. https://doi.org/10.5565/rev/educar.1117

Cortés González, P., González Alba, B. y Sánchez Mesa, F. (2018). Agrupamientos escolares y retos para la educación inclusiva en infantil y primaria. Tendencias Pedagógicas, 32, 75-90. https://doi.org/10.15366/ tp2018.32.006

Crisol, E. (2019). Hacia una educación inclusiva para todos. Nuevas contribuciones. Profesorado, Revista de Currículum y Formación del Profesorado, 23(1), 1-9.

De Boer, A., Pijl, S. y Minnaert, A. (2011). Regular primary schoolteachers' attitudes towards inclusive education: A review of the literature. International Journal of Inclusive Education, 15(3), 331-353. https://doi.org/10.1080/13603110903030089

Del Rincón, D., Arnal, J., Latorre, A. y Sans, A. (1995). Técnicas de investigación en ciencias sociales. Dykinson.

Domingo, J. (2008). El aprendizaje cooperativo. Cuadernos de Trabajo Social, 21, 231-246.

Duk, C. y Murillo, F. J. (2016). La inclusión como dilema. Revista Latinoamericana de Educación Inclusiva, 1O(1), 11-14. https://doi.org/10.4067/So718-73782016000100001

Dyson, B., Colby, R. y Barratt, M. (2016). The co-construction of cooperative learning in physical education with elementary classroom teachers. Journal of Teaching in Physical Education, 35(4), 370-380. http://doi/10.1123/jtpe.2016-0119

Escudero, J. M. y Martínez, B. (2011). Educación inclusiva y cambio escolar. Revista Iberoamericana de Educación, 55(1), 85-105. https://doi.org/10.35362/rie550526

Jenkins, J. R., Antil, L. R., Wayne, S. K. y Vadasy, P. F. (2003). How cooperative learning works for special education and remedial students. Exceptional Children, 69(3), 279-292. https://doi.org/10.1177/001440290306900302

Flick, U. (2004). Introducción a la investigación cualitativa. Morata.

Gewerc, A. y Vázquez-Calvo, B. (2020). Etnografía en la era (pos)digital. En J. M. Sancho, F. Hernández, L. Montero, J. Pons, J. I. Rivas y A. Ocaña (Coords.), Caminos y derivas para otra investigación educativa y social (pp. 181-194). Octaedro.

Gillies, R. M. (2014). Cooperative learning: Developments in research. International Journal of Educational Psychology, 3(2), 125-140.

Gillies, R. M. (2016). Aprendizaje cooperativo: Revisión de la investigación y la práctica. Australian Journal of Teacher Education, 41(3), 39-54. https://doi.org/10.14221/ajte.2016v41n3.3 
González-Alba, B. (2020). Breakout Educativo. Otra forma de aprender. En E. López-Meneses, D. Cobos-Sanchis, L. Molina-García, A. Jaén-Martínez, y A. H. Martín-Padilla (Eds.). Claves para la innovación pedagógica ante los nuevos retos. Respuestas en la vanguardia de la práctica educativa (pp. 346-352). Octaedro Editorial.

González-Alba, B., Cortés González, P. y Mañas Olmo, M. (2020). La inclusión educativa através de la narrativa-biográfica. Experiencias del profesorado de pedagogía terapeútica. Revista Infancia, Educación y Aprendizaje, 6(1), 19-36.

Hernández Rivero, V. M., Casillas, S., Cabezas, M. y Basilota, V. (2020). La investigación participativa y colaborativa. En J. M. Sancho, F. Hernández, L. Montero, J. Pons, J. I. Rivas y A. Ocaña (Coords.). Caminos y derivas para otra investigación educativa y social (pp. 139- 152). Octaedro.

Hijzen, D., Boekaerts, M. y Vedder, P. (2007). Exploring the links between students' engagement in cooperative learning, their goal preferences and appraisals of instructional conditions in the classroom. Learning and Instruction, 17(6), 673-687.

https://doi.org/10.1016/j.learninstruc.2007.09.020

Iglesias Muñiz, J. C., López Miranda, T. y Fernández-Río, J. (2017). La enseñanza de las matemáticas a través del aprendizaje cooperativo en $2^{\circ}$ curso de educación primaria. Contextos Educativos, 2, 47-64. http://doi.org/10.18172/con.2926.

Iglesias Rodríguez, A., García Riaza, B. y Sánchez Gómez, M. C. (2017). Collaborative learning and mobile devices: An educational experience in primary education. Computers in Human Behavior, 72, 664-677. https://doi.org/10.1016/j.chb.2016.07.019

Jarauta, B. y Bozu, Z. (2013). Portafolio docente y formación pedagógica inicial del profesorado universitario: Un estudio cualitativo en la universidad de Barcelona. Educación XXI: Revista de la Facultad de Educación, 16(2), 343-361. https://doi.org/10.5944/educxx 1.2.16.10345

Johnson, D. W., Johnson, R. T. y Holubec, E. J. (2013). Cooperation in the classroom. Interaction Book Company.

Lata, S. y Castro. M. M. (2015). El aprendizaje cooperativo, un camino hacia la inclusión educativa. Revista Complutense de Educación, 27(3), 1085-1101.

https://doi.org/10.5209/rev_rced.2016.v27.n3.47441

Loor, L.R; Palma, M. M., Saltos, L. J y Bolívar, O. E. (2018). El aprendizaje cooperativo como una estrategia de enseñanza del idioma de inglés en las escuelas públicas del Ecuador. Revista Científica Dominio de las Ciencias, 4(3) 431-448. https://doi.org/10.23857/dc.v4i3.817

Martínez Lirola, M. (2016a). Hacia una resolución efectiva de conflictos en las aulas universitarias: Ejemplos a través del debate cooperativo. Zona Próxima, 24, 103-114. https://doi.org/10.14482/ zp.24.8724

Martínez Lirola, M. (2016b). How to use cooperative learning for assessing students' emotional competences: a practical example at the tertiary level. Profile, 18(2), 153-165.

https://doi.org/10.15446/profile.v18n2.52593

Mendo-Lázaro, S., León-del-Barco, B., Felipe-Castaño, E., Polo-del-Río, M. I. e Iglesias-Gallego, D. (2018). Cooperative team learning and the development of social skills in higher education: The variables involved. Frontiers in Psychology, 9, art 1536. https://doi.org/10.3389/fpsyg.2018.01536

Montanero, M. (2019). Didáctica general. Planificación y práctica de la enseñanza primaria. Servicio de publicaciones de la Universidad de Extremadura.

Montanero, M. y Tabares, C. (2020). El aprendizaje cooperativo en la educación primaria: Un estudio sobre el pensamiento del profesorado y la práctica docente en Extremadura. 
Profesorado. Revista de Currículum y Formación del Profesorado, 24(3), 357-379.

https://doi.org/10.30827/profesorado.v24i3.8200

Moriña, A. (2011). Aprendizaje cooperativo para una educación inclusiva: Desarrollo del programa PAC en un aula de educación primaria. ESE: Estudios sobre Educación, 21, 199-216.

Orozco, I. y Moriña, A. (2019). Prácticas docentes para una pedagogía inclusiva en educación primaria: Escuchando las voces del profesorado. Aula Abierta, 48(3), 331-338. https://doi.org/10.17811/rifie.48.3.2019.331-338

Polo, M. I., Mendo, S., Fajardo, F. y León, B. (2017). Una intervención en aprendizaje cooperativo sobre el perfil del observador en la dinámica bullying. Universitas Psychologica, 16(1), 1-13. https://doi.org/10.11144/Javeriana.upsy 16-1.iacp

Pons, R. M., González-Herrero, M. E. y Serrano, J. M. (2008). Aprendizaje cooperativo en matemáticas: Un estudio intracontenido. Anales de Psicología, 24(2), 253-261.

Prieto Saborit, J. A. y Nistal Hernández, P. (2009). Influencia del aprendizaje cooperativo en educación física. Revista Iberoamericana de Educación, 49(4), 1-8.

Pujolás, P. (2008). El aprendizaje cooperativo como recurso y como contenido. Aula de Innovación Educativa, 170, 37-41.

Pujolás, P. (2012). Aulas inclusivas y aprendizaje cooperativo. Educatio Siglo XXI, 30, 89-112.

Pujolás, P., Lago, J. R. y Naranjo, M. (2013). Aprendizaje cooperativo y apoyo a la mejora de las prácticas inclusivas. Revista de Investigación en Educación, 11(3), 207-218.

Ribosa, J. y Durán, D. (2017). Cooperación, juego y matemáticas: Análisis de la aplicación del Tridio cooperativo con alumnado de primaria. Revista de Investigación en Didáctica de la Matemática, $11(3), 205-231$.

Riera, G. (2011). El aprendizaje cooperativo como metodología clave para dar respuesta a la diversidad del alumnado desde un enfoque inclusivo. Revista Lationamericana de Educación Inclusiva, 5(2), 113-149.

Ruiz Olabuénaga, J. I. (1999). Metodología de la investigación cualitativa. Universidad de Deusto.

Santos, M., Lorenzo, M. M., Godás, A. O. y Sotelino, A. L. (2020). Aprendizaje cooperativo, autoimagen y percepción del ambiente de aprendizaje en educación secundaria. Bordón. Revista de Pedagogía, 72(4), 117-132. https://doi.org/10.13042/Bordon.2020.77726

Sharan, Y. (2010). Cooperative learning for academic and social gains: Valued pedagogy, problematic practice. European Journal of Education, 45(2), 300-313. https://doi.org/10.1111/j.1465-3435.2010.01430.x

Strauss, A. y Corbin, J. (2002). Codificación axial. En A. Strauss y J. Corbin (Eds.), Bases de la investigación cualitativa (pp. 134-156). Universidad de Antioquia.

Surian, A. y Damini, M. (2014). "Becoming" a cooperative learner-teacher. Anales de Psicología, 3O(3), 808-817. https://doi.org/10.6018/analesps.30.3.201521

Trujillo, F. (2002). Aprendizaje cooperativo para la enseñanza de la lengua. Publicaciones, 32, 147162.

Valdebenito, V. y Durán, D. (2015). Formas de interacción implicadas en la promoción de estrategias de comprensión lectora a través de un programa de tutoría entre iguales. Revista Latinoamericana de Psicología, 47(2), 75-85. https://doi.org/10.1016/j.rlp.2014.07.001

Veldman, M. A., Van Kuijk, M. F., Doolaard, S. y Bosker, R. J. (2020). The proof of the pudding is in the eating? Implementation of cooperative learning: differences in teachers' attitudes and 
beliefs. Teachers and Teaching, 26(1), 103-117.

https://doi.org/10.1080/13540602.2020.1740197

Webb, N. (2009). The teacher's role in promoting collaborative dialogue in the classroom. British Journal of Educational Psychology, 79, 1-28. https://doi.org/10.1348/000709908X380772

\section{Breve CV de los/as autores/as}

\section{Blas González Alba}

Diplomado en Educación Musical y Licenciado en Psicopedagogía por la Universidad de Málaga, Licenciado en Antropología Social y Cultural por la UNED, Máster y Experto en Atención Temprana y Máster en Psicopedagogía Clínica. Máster en Cultura de Paz, derechos humanos y Educación, doctor en Ciencias de la Educación por la Universidad de Málaga y maestro de Pedagogía Terapéutica para la Junta de Andalucía. Desde el año 2014 es miembro activo del grupo de investigación consolidado ProCIE de la Universidad de Málaga en el que colabora en tareas de investigación en el marco de los diferentes proyectos en los que participa el grupo, durante los últimos años gran parte de su producción científica está relacionada con el ámbito de la atención a la diversidad, la inclusión, resiliencia y la investigación narrativa biográfica. Email: blas@uma.es

ORCID ID: https://orcid.org/0000-0002-4769-6522

\section{Pablo Cortés González}

Profesor Titular del Departamento de Didáctica y Organización Escolar de la Universidad de Málaga. Miembro del grupo de investigación consolidado ProCIE -HUM619- de la Junta de Andalucía y de la British Sociological Association y coordinador del Máster en profesorado en Educación Secundaria Obligatoria y Bachillerato, Formación Profesional y Enseñanza de idiomas. También participa activamente en redes académicas a nivel nacional e internacional. Sus líneas de investigación versan en torno a las narrativas, la identidad profesional de los agentes educativos, la resiliencia, las prácticas disruptivas y las ecologías del aprendizaje, teniendo un centenar de artículos y capítulos de libro sobre dichas temáticas y forma parte de las Redes de investigación: RETINDE, REUNID, RIDIPD. Email: pcortes@uma.es

ORCID ID: https://orcid.org/0000-0002-9604-044X

\section{Analia Leite Méndez}

Docente e investigadora con más de 25 años de experiencia. Hasta el año 2004 en la Facultad de Humanidades de la Universidad Nacional del Nordeste (Chaco) Argentina y desde el 2004 hasta la fecha en la Universidad de Málaga. Doctora en Educación. Profesora Titular del Departamento de Didáctica y Organización Escolar de la Universidad de Málaga. Integra el Grupo de Investigación ProCIE (Grupo consolidado de la Junta de Andalucía). Es autora de numerosas publicaciones especializadas y ponente en actos académicos y educativos tanto nacionales como internacionales. Investigadora del Grupo Procie (HUM619) Autora y coautora de artículos y textos en el ámbito de la formación del profesorado, identidades docentes, voluntariado y formación docente desde una perspectiva narrativa-biográfica. Email: aleite@uma.es

ORCID ID: https://orcid.org/0000-0001-5064-999X 